\title{
Structure Design and Simulation of Plug-seedling Gripping Device Cao Lifang ${ }^{1, a}$, Liu Cunxiang ${ }^{1, \text { a* }}$ and Zhang Chuangchuang ${ }^{1, a}$ \\ ${ }^{1}$ College of Mechanical and Electrical Engineering, Agricultural University of Henan, Zhengzhou, 450002, China \\ a15038041749@139.com
}

Keywords: Transplanting of plug-seedling, Gripping device, Cam indexers, Motion simulation.

\begin{abstract}
Transplanting of plug-seedling is an important method for seedling transplanting. Considering the problems existing in the transplanting of plug-seedling, a new plug-seedling gripping device based on mechanical finger was designed using Solidworks in this paper. The device controlled the rotation and stop of four fingers using cam indexers to accomplish the capture, transporting and delivery of plug-seedlings. Then the model data was imported into the simulation software of ADAMS by using the conversion interface. The motion simulation of the model capturing, transporting and delivering of plug-seedlings was performed accurately and reliably through ADAMS.
\end{abstract}

\section{Introduction}

The strong and unified seedling is expected for crops cultivation to reduce managing cost, as well as desired for the automatic machine to graft and transplant [1-4]. Transplanting of plug-seedling is an important measure for high yield crop by making full use of sunlight, gaining more farming time and preventing natural disasters. But at present, the transplanting machinery is mainly semi-automatic transplanting machine. Semi-automatic transplanting still needs getting seedlings artificially. Due to the limiting speed of artificial feeding seedling and the need of more workers, this not only wastes manpower, reduces the efficiency of work, but also increases the machine loading greatly for additional devices. So the semi-automatic transplanting machine is not suitable for completing a large area of transplanting in short period of time [5].

In order to solve the problem of labor waste, low production efficiency and the difficultly to realize large area operation, the full-automatic machine for plug-seedlings transplanting will be the trend in the future. Reliable gripping device is the key to realize the automatic transplanting. Existing transplanting machines are designed for transplanting of pot seedling or bare-rooted seedling. Therefore they cannot be applied to new type of seedling- transplanting of plug-seedling [6]. Therefore this paper designed a kind of machine for transplanting of plug-seedling through the three-dimensional model software of Solidworks and the kinematics simulation and analysis were performed through the virtual simulation software of ADAMS.

\section{Design and Working Principle of Gripping Mechanism}

Design of Plug-Seedling Gripping Mechanism. As shown in Fig. 1, the gripping machine mainly consisted of a cam indexers, the upper and the lower plates, mechanical fingers, locking device, block etc. The function was achieved through the motion of the cam indexers and the locking device. The cam indexers controlled the mechanical fingers to grip and lift the seedlings. Then the cam indexer rotated to drive seedlings, put the seedlings at the position of the tube and performed the lifting movement. The locking device controlled the relative position between the upper and lower plates to achieve the locking and unlocking motion of the pointer. When the fingers gripped the seedlings, the seedlings would be locked in mechanical fingers in fear of falling off in transit. When the seedlings reached the tube, the device loosened seedlings. Then the seedlings would fall into the seedling tube. 


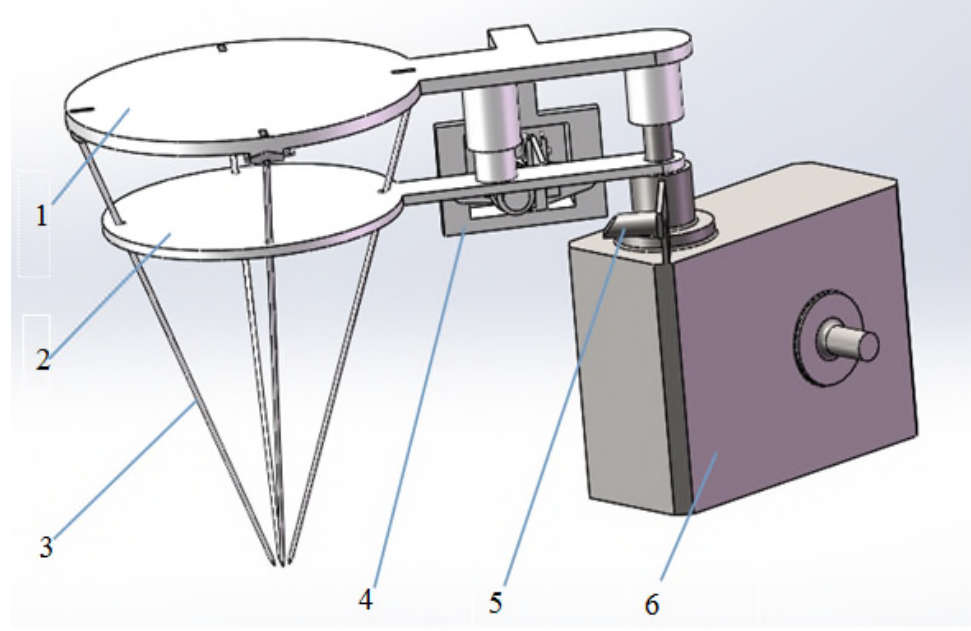

Fig. 1 Structure of the gripping device.

1. Upper plate 2. Lower plate 3. Needle 4. Locking device 5. Block 6. Cam indexer

Working Principle of the Seedling Mechanism. Working principle is as follows. The initial position of fingers was above the seedlings. When the motor began to drive 6 to work, 1 fixed to the output shaft start downward movement. 3 were controlled by 2 to rotate to the center, until it got to the bottom of the stroke with the tilt angle of 71 degree. Then the seedlings were gripped. At the same time, 1 fixed on 4 started to fall to the bottom. Then the gripping motion was completed as 2 and 1 are locked together. After gripping the seedlings, 1 and 2 rose to the top of stroke with the output shaft of indexers. Then they rotated together with the shaft to top of the tube, and dropped to the bottom of the stroke. The sending motion was completed. When the seedlings reached the bottom, 5 touched the button of 4 , then 1 and 2 were separated. 1 moved upward with the output shaft of indexers and 3 opened. The putting motion was completed as the seedlings dropped.

Cam Indexers. The cam indexers was the mechanism to realize intermittent motion with advantage of high indexing precision, steady operation and great transmission torque, positioning self-locking, compact structure, small volume, low noise, good performance at high speed, long lifespan, etc. It transferred continuous rotation to intermittent rotation, swing, lift and other motions. It was mainly used for automatic processing, assembly, testing and other equipment. In order to satisfy the movement of stopping gripping, rotation and delivery, this thesis chosen the lifting and swinging cam indexers which its output shaft could perform reciprocating motion up and down, intermittent rotation and swing motion. The action sketch is shown in Fig. 2.

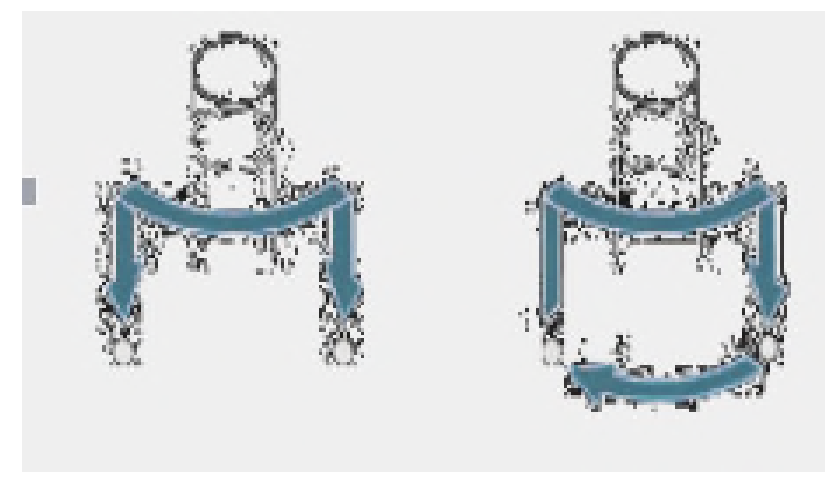

Fig. 2 Action sketch map.

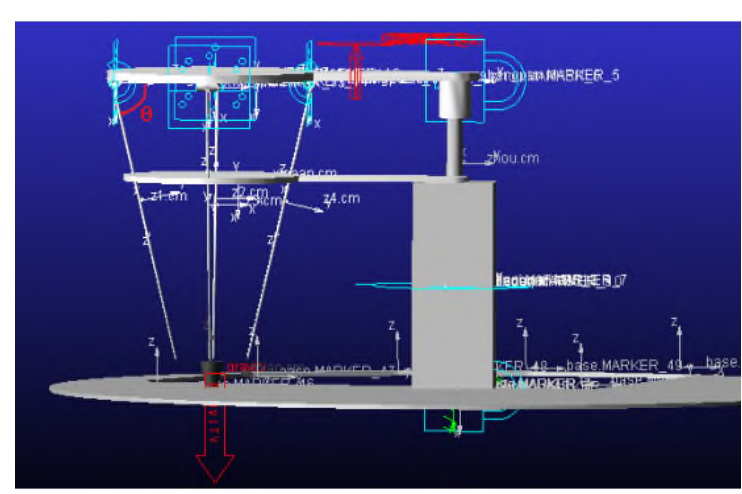

Fig. 3 The simplified model diagram.

The cam indexers was composed of a cam and a rotary tower, which was suitable for the left and right swing, the upper and lower clamping systems and the conveying in high precision and high speed. It provides various forms of intermittent movement. The largest movement itinerary was $60 \mathrm{~mm}$, which was sufficient to cover the height of the tray. 


\section{The Model Simulation}

Model Data Adding. Three-dimensional solid models were established and parameter calculation of the mechanical components was performed using Solidworks. Then the model was transferred into ADAMS to carry out the movement simulation analysis. $+Z$ was specified as the direction of gravity acceleration. In order to make the virtual prototype simulate the moving structure quickly and clearly, some parts that didn't need analyzing or didn't affect the overall effect of simulation components were omitted. The function of these parts can be replaced by adding constraints, friction, which not only could shorten the simulation time, but also could improve the success rate of simulation [7-8]. Diagram of the simplified model is shown in Fig. 3.

Add Driver. Upper plate and lower plate are the key components used to connect the cam indexer and the needles. They were used not only to complete the motion of rise, fall, rotation, but also to control seedling needles to rotate. The goal of these motions was to control needles to open and close. Then gripping and releasing of seedlings were achieved. So their motions were particularly important. A study based on the schedule of $40 \mathrm{~mm}$ was done in this paper.

The driving function of the upper plate was:

$$
\begin{aligned}
& f(\text { time }) \\
& =\text { step }(\text { time }, 0,0,0.2,40) \\
& + \text { step }(\text { time }, 0.2,0,0.4,-40) \\
& + \text { step }(\text { time }, 0.5,0,0.7,40) \\
& + \text { step }(\text { time }, 0.7,0,0.9,-40)
\end{aligned}
$$

The driving function of the lower plate was:

$$
\begin{aligned}
& f(\text { time }) \\
& =\operatorname{step}(\text { time }, 0.2,0,0.4,-40) \\
& + \text { step }(\text { time }, 0.5,0,0.7,40) ;
\end{aligned}
$$

The driving function of synchronous rotation was :

$$
\begin{aligned}
& f(\text { time }) \\
& =\text { step }(\text { time }, 0.4,0,0.5,90 d) \\
& + \text { step }(\text { time }, 0.9,0,1.0,-90 d)
\end{aligned}
$$

According to the driving function, driving function curves of displacement and rotation of upper plate and lower plate are shown in Fig. 4.

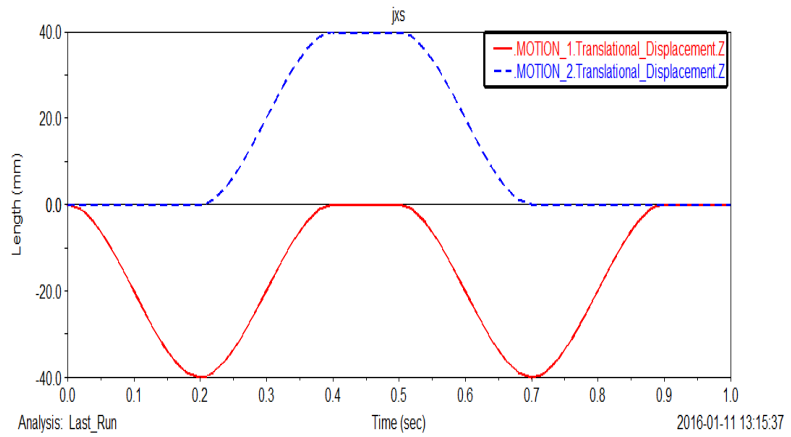

(a) Motion 1 and motion 2

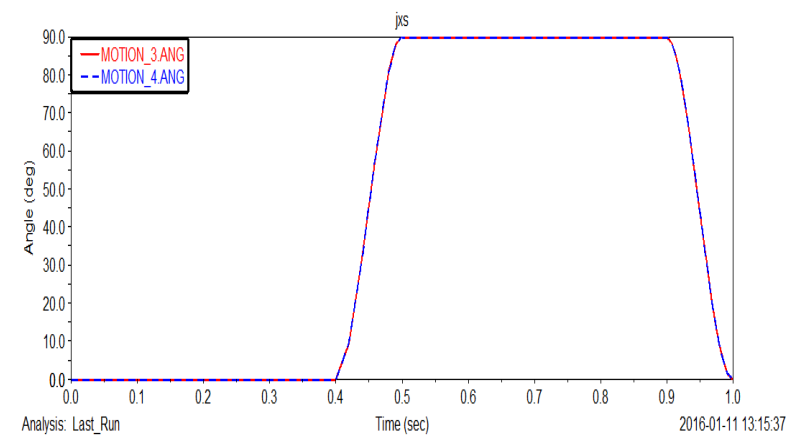

(b) Motion 3 and motion 4

Fig. 4 Drive function curve. 
From the above curves, from 0 to $0.2 \mathrm{sec}$, the upper plate moved $40 \mathrm{~mm}$ along the $+Z$; from 0.2 to $0.4 \mathrm{sec}$, upper plate and lower plate simultaneously moved $40 \mathrm{~mm}$ along the $-\mathrm{Z}$ axis; from 0.4 to 0.5 sec, upper plate and lower plate simultaneously rotated 90 degrees clockwise; from 0.5 to $0.7 \mathrm{sec}$, upper plate and lower plate simultaneously moved $40 \mathrm{~mm}$ along the $+Z$; from 0.7 to $0.9 \mathrm{sec}$, upper plate moved $40 \mathrm{~mm}$ along the $-\mathrm{Z}$ axis; from 0.9 to $1.0 \mathrm{sec}$, upper plate and lower plate simultaneously rotated 90 degrees counter-clockwise.

Motion Analysis. The four needles rotated synchronously with the change of the relative displacement of the upper plate and lower plate. They could clamp and release the seedlings according to the change of the angle $\theta$ between the upper plate and every needle. The $\theta$ was marked in Fig. 3. The measured angle $\theta$ is shown in Fig. 5.

As shown in Fig. 5, from 0 to $0.2 \mathrm{sec}$, upper plate moved along the $+Z$ and lower plate kept still. At the same time, needles gathered toward the center. And the $\theta$ changed from 78.5 degrees to 71 degrees. From 0.2 to $0.7 \mathrm{sec}$, upper plate and lower plate were locked, the needles were relatively static and the $\theta$ remained the same. After 0.7 seconds, upper plate moved along the $-Z$ axis. Then needles opened and the $\theta$ restored from 71 degrees to 78.5 degrees.

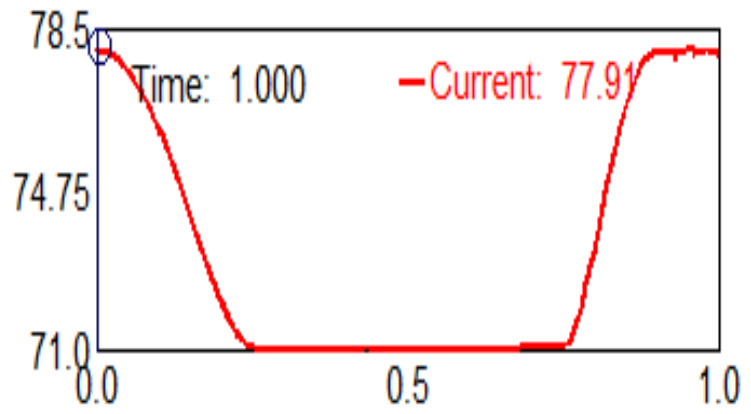

Fig. 5 The curve of angle.

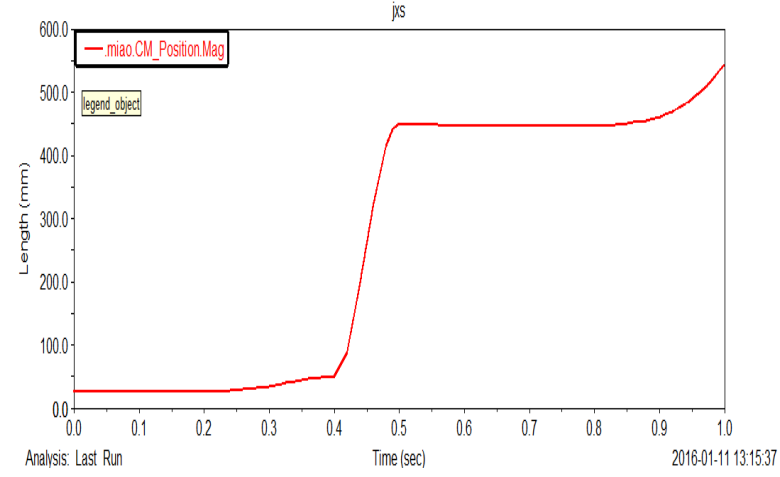

Fig. 6 The curve of seedling movement.

Through the relative motion of the upper plate and lower plate, and the synchronous rotation of four needles, seedlings moved under the driving of the needles. The displacement curve is shown in Fig. 6. The trajectory of a seedling marked in the center of mass with the red line is shown in Fig. 7.

The trajectory of the seedlings mainly consisted of two parts. Firstly, the motion of gripping and sending was achieved through plug-seedling gripping device. Secondly, the seedlings dropped from mechanical fingers based on gravity. The two parts of the motion function are shown below:

The function before 0.7 seconds along the $\mathrm{Z}$ axis is:

$$
\begin{aligned}
& f(\text { time })=\operatorname{step}\left(t, t_{0}, h_{0}, t_{1}, h_{1}\right) \\
& f(\text { time })=\left\{\begin{array}{l}
h_{0}, t \leq t_{0} \\
h, t_{0}<t<t_{1} \\
h_{1}, t \geq t_{1}
\end{array}\right.
\end{aligned}
$$

The displacement function after 0.7 seconds along the $\mathrm{Z}$ axis is:

$$
S=4903.3 t^{2}-7315.2 t+2717, t=[0.7,1.0)
$$

The movement curve of the seedling along the $\mathrm{Z}$ axis through the virtual simulation software of ADAMS is shown in Fig. 8. The differential solution of displacement function was calculated. The result is shown as a red dashed line in Fig. 8. As shown in Fig. 8, the red dashed line and the velocity function curve was basically coincident. That just coincided with the rule that the derivative of the displacement is speed. 


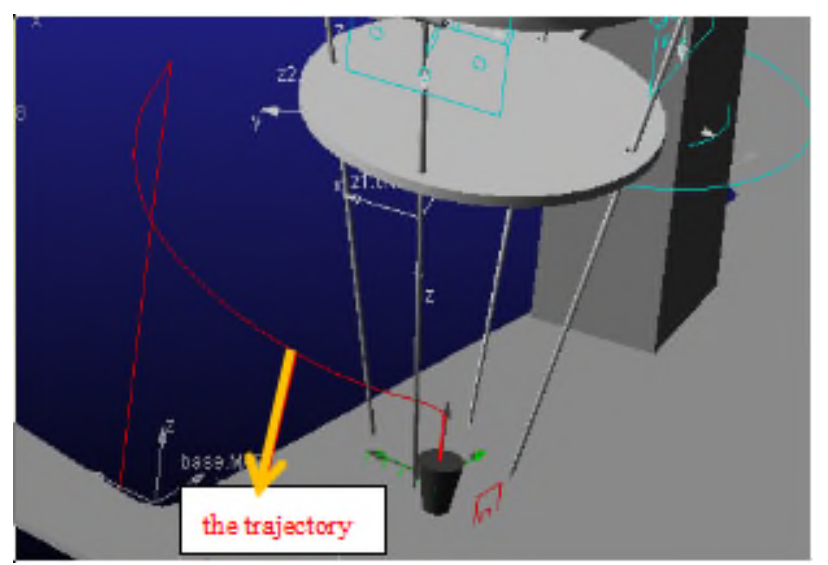

Fig. 7 The trajectory of seedling.

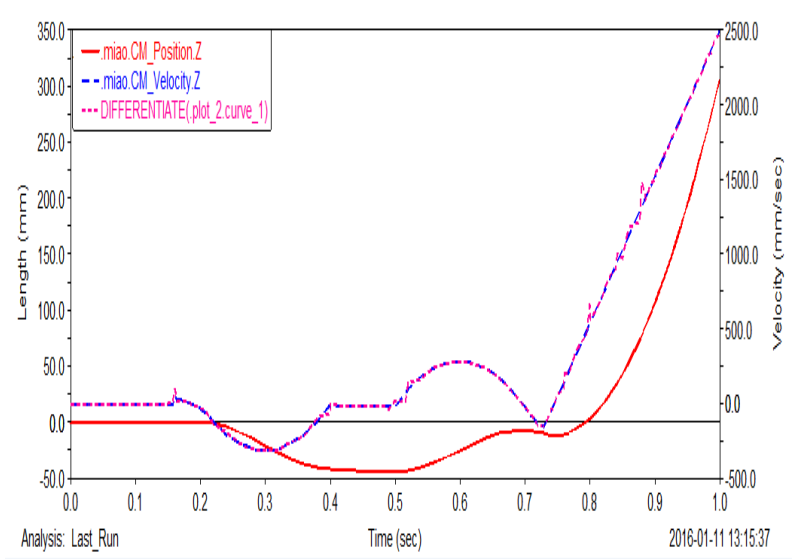

Fig. 8 The movement curve of seedling.

\section{Conclusion}

Design and simulation analysis of plug-seedling gripping device was done in the paper. The results showed that the plug-seedling gripping device could accomplish the capture, transporting and delivery of plug-seedlings accurately and reliably. The model was simulated and analyzed through the virtual analysis software ADAMS. The optimal structure was obtained quickly without making the actual model and the artificial calculation of complex situations. Through these methods, the development time and designing cost were reduced greatly.

\section{Acknowledgement}

In this paper, the research was sponsored by the Agricultural Scientific and Technological Production Funds Project of China (Project No. 2009GB2D000214).

\section{References}

[1] S. J. Yi, Y. F. Liu, C. Wang, G. X. Tao, H. Y. Liu and R. H. Wang, Experimental study on the performance of bowl-tray rice precision seeder, Int. J. Agric. Biol. Eng. 7(1) (2014) 17-25.

[2] K. Z. Tan, Y. H. Chai, W. X. Song, X. D. Cao, Identification of diseases for soybean seeds by computer vision applying BP neural network, Int. J. Agric. Biol. Eng. 7(3) (2014) 43-50.

[3] Q. C. Feng, C. J. Zhao, K. Jiang, P. F. Fan and X. Wang, Design and test of tray-seedling sorting transplanter, Int. J. Agric. Biol. Eng. 8(2) (2015) 14-20

[4] Y. Li, C. Liu and T. Zhang, Design and experiment of vegetable grafting machine with double manipulators, T. Chinese Soc. Agr. Mach. 40(9) (2009) 175-181.

[5] W. Cui, P. Xu, H. F. Wang and G. F. Li, et al. Present Status and Analysis of Day -land Auto-transplanting Seeding Technique, J. Agr. Mechanization Res. 6 (2015) 1-5, 28.

[6] W. Q. Ding, H. P. Mao, J. P. Hu and F. Liu, Structure Design and Simulation Analysis on the Plug Seedlings Auto-Transplanter, J. Agr. Mechanization Res. 10 (2011) 75-77, 137.

[7] Q. Ma, Z. Chen, X. C. Zhang and Xiaoan Hu, Motion Simulation Analysis of Harvesting Robot Arm Based on ADAMS. J. Agr. Mechanization Res. (5) (2013) 7-40, 44.

[8] H. X. Li, H. Li and G. J. Xu, et al. Virtual Prototype Simulation Analysis for Main Parts of Excavators. Eng. Mach. 40(10) (2009) 36-39. 